\title{
Lateral patellar luxation in nine small breed dogs
}

\author{
F. Di Dona ${ }^{1, *}$, G. Della Valle ${ }^{1}$, C. Balestriere ${ }^{1}$, B. Lamagna ${ }^{1}$, L. Meomartino ${ }^{2}$, G. Napoleone ${ }^{1}$, F. Lamagna ${ }^{1}$ and \\ G. Fatone ${ }^{1}$ \\ ${ }^{I}$ Department of Veterinary Medicine and Animal Productions, University of Napoli "Federico II", Italy \\ ${ }^{2}$ Interdepartmental Center of Veterinary Radiology, University of Napoli "Federico II", Italy
}

\begin{abstract}
The objective of this paper was to describe the clinical features, the management and the outcome of nine small breed dogs affected with lateral patella luxation referred during the period between January 2010 and December 2014. Patellar luxations were classified according to: breed, age, sex, weight, and grade of patellar luxation, as well as if unilateral or bilateral, and concurrent cranial cruciate ligament lesion. In affected dogs, surgical correction consisted in the combination of tibial tuberosity transposition and soft tissue procedure. Adjunctive condroplasty or trochleoplasty was performed as needing. The outcome was found positive after surgical management with low complication rate and complications have been easily managed with high success rate.
\end{abstract}

Keywords: Dog, Lateral, Luxation, Patellar.

\section{Introduction}

Patellar luxation (PL) is one of the most common orthopedic disease in dogs, and can affect both large and small dogs and may be seen in cats as well (Nunamaker, 1985; DeAngelis, 1996; Ness et al., 1996; Linney et al., 2011). Lateral patellar luxation (LPL) occurs less frequently than does medial patellar luxation (MPL) (Roush, 1993). LPL is commonly diagnosed in large or giant breed dogs, especially the St. Bernard, while it seems to be an unusual occurrence in small breed dogs (Roush, 1993; Hayes et al., 1994; LaFond et al., 2002; L'Eplattenier and Montavon, 2002; Harasen, 2006). In a recent study, which evaluated retrospectively $65 \mathrm{dogs}$ of all breeds with LPL, medium and large breed dogs were more affected; and less than $10 \%(6 / 65)$ of all dogs were small breed dogs (Kalff et al., 2014).

The purpose of this paper is to describe clinical presentation, radiographic findings, management and outcome of 9 small breed dogs with a diagnosis of LPL.

\section{Materials and Methods}

Medical records of small breed dogs with confirmed diagnosis of LPL admitted to the Veterinary Teaching Hospital at the University of Napoli (2010-2014) were identified. According to Kennel Club standards for weight, dogs with a physical weight less than $9 \mathrm{~kg}$ were considered small breed (Priester, 1972; Hayes et al., 1994; Bound et al., 2009). Data collection included: breed, age, sex, weight, grade of PL, as well as if unilateral or bilateral, and concurrent cranial cruciate ligament $(\mathrm{CrCL})$ lesion. Grade of PL was determined by referencing the description of PL to a simplified grading system by Roush (1993). Grade I: Patella can be manually luxated but returns to normal position when released. Grade II: Patella luxates with stifle flexion or on manual manipulation and remains luxated until stifle extension or manual replacement occurs. Grade III: Patella luxated continually, and can be manually replaced but will reluxate spontaneously when manual pressure is removed. Grade IV: Patella luxated continually and cannot be manually replaced. Orthopedic examination consisted in gait evaluation by visual inspection, joint flexion-extension movements and range of motion evaluation, and grade of PL. Radiographic images in both medio-lateral and craniocaudal projections were taken. All dogs were treated surgically and were evaluated at clinical follow-up.

\section{Surgery}

All surgical procedures were executed throughout a cranio-medial, parapatellar, incision to the stifle joint. The surgical techniques used in dogs with LPL were grouped in soft tissue procedures (STP) and osseous procedures (medial tibial tuberosity transposition [TTT]; femoral recession trochleoplasty or chondroplasty). The soft tissue techniques included lateral retinacular release and medial imbrication (Roush, 1993); the deep fascia was released laterally and imbricated medially in order to produce slight medial tension. The osseous procedures included TTT, femoral recession trochleoplasty (TP) or chondroplasty (CP). TTT consisted in osteotomy of the tuberosity with intact distal attachment; tibial crest was then moved medially a sufficient distance to provide straight alignment of the quadriceps mechanism and was stabilized with two Kirschner wires $(0.8$ to $1.5 \mathrm{~mm}$; Alcyon Italia S.p.A., Italy) without using tension band

*Corresponding Author: Francesco Di Dona. Department of Veterinary Medicine and Animal Productions, University of 
fixation. Femoral recession trochleoplasty was performed when the trochlear sulcus was shallow or absent; trochlear block recession was performed through elevating an osteochondral block from the patellar groove, removing bone from the bottom of the incised block to deepen the sulcus, and then replace the osteochondral block (Priester, 1972; Bound et al., 2009). In young dogs (less than 8 months) chondroplasty was performed by elevating the hyaline cartilage and removing subcondral bone beneath the cartilage flap (Roush, 1993). The adjunctive surgical technique used in dogs with concurrent CrCL injury consisted in the medial and lateral fabellae suturing (FS) (DeAngelis and Lau, 1970; Shaver et al., 2014). Two unilateral-session surgeries were performed in dogs bilaterally affected.

\section{Pre- and post-operative care}

All dogs were premedicated with intramuscular administration of methadone $(0.3 \mathrm{mg} / \mathrm{kg}$; Eptadone: $\mathrm{L}$. Molteni \& C. S.p.A., Italy) or morphine $(0.4 \mathrm{mg} / \mathrm{kg}$; Morfina cloridrato: L. Molteni \& C. S.p.A., Italy) and dexmedetomidine $(5 \mu \mathrm{g} / \mathrm{kg}$; Dexdomitor: Vetoquinol Italia S.r.l., Italy). Intravenous administration of propofol (4-6 mg/kg; Propovet: Esteve S.p.A., Italy) was used to induce anesthesia, then maintained via gaseous mixture of oxygen and isoflurane (Isoflurane Vet: Merial Italia S.p.A., Italy). Peripheral sciatic and femoral nerve block with lidocaine ( $2 \mathrm{mg} / \mathrm{kg}$; Lidocaina Cloridrato: A.T.I. S.r.l., Italy) was obtained. Perioperative antibiotic therapy included intravenous administration of cephazolin (22 mg/kg; Cefazolina Teva: Teva Italia S.r.1., Italy). Postoperative analgesia included intramuscular administration of methadone (0.3 mg/kg; Eptadone: L. Molteni \& C. S.p.A., Italy) as required for up to $48 \mathrm{~h}$ and either carprofen $(2 \mathrm{mg} / \mathrm{kg}$ SID; Rimadyl: Pfizer Italia S.r.l., Italy) or firocoxib (5 mg/kg SID; Previcox: Merial Italia S.p.A., Italy) for 6 to 10 days following surgery. Dogs were dismissed from the hospital 24-48h following surgery.

\section{Outcome and Complications}

Clinical follow was performed for each dog at 7 and 14 days, then at 4 to 12 weeks following surgery, and thereafter by telephone interview. The outcome of surgical correction was classified, according to dogs' status, in: excellent (no gait abnormality), good (mild or intermittent gait abnormality), fair (moderate gait abnormality), and poor (severe gait abnormality). Frequency and type of postoperative complication associated with LPL stabilization were registered. Dogs that underwent a revision surgery were considered as having a major complication. Whereas complication did not required additional treatments were classified as minor.

\section{Statistical analysis}

All statistical analyses were performed using commercial software (Prism GraphPad Software, Inc,
USA). Descriptive statistics were calculated and data were reported as median and range. Categorical data were expressed as frequencies. Statistical power was inadequate to compare outcome measures or incidence of complications with signalment or surgical variables.

\section{Results and Discussion}

Of the one hundred thirty-seven dogs with a diagnosis of PL in the reference period, LPL was present in fourteen dogs. However only nine of them met the inclusion criteria and were classified as small breed dogs. Seven dogs were female (one neutered) and the remaining two were intact male. Dogs ranged in age from 5 to 132 months (median $=18$ months). Body weight ranged from 1.3 to $8.8 \mathrm{~kg}$ (median $=4 \mathrm{~kg}$ ). The following breed were registered: Poodle $(n=3)$, Pinscher $(n=2)$, Pekingese, Yorkshire, Cavalier King Charles Spaniel and mixed-breed. Five dogs had a diagnosis of grade III luxation and one of them was bilaterally affected. The remaining four dogs were equally affected with grade I and II luxation, whereas none of the dogs had a diagnosis of a grade IV luxation. One dog had a history of vehicular trauma, while another one experienced an acute lameness due to a CrCL injury.

Surgical correction was performed in 10 stifles and consisted in TTT and STP for all the dogs included. Two dogs received adjunctive chondroplasty because of their young age, whereas in other three dogs, the deepening of the trochlear groove was obtained through a block recession trochleoplasty. One dog was managed in a single session surgery for both LPL and CrCL injury, through medial TTT and subsequently FS. Major complications were observed only in two dogs that experienced a lateral reluxation, and were managed with a second soft tissue procedure to improve medial tension. Two dogs had implant migration and concomitant seroma, and were considered as having a minor complication. Follow-up was performed for all dogs. Four dogs with five stifles treated were classified as having excellent outcome; three dogs as having good outcome; and two dogs as having fair outcome. These last two dogs with moderate gait abnormalities at last examination were both affected by concurrent injuries (CrCL injury; aftereffects of a pelvic trauma) (Table 1). PL is a common stifle disorder in dogs, and MPL occurs more frequently than does LPL, especially in small breed dogs (Nunamaker, 1985; Vasseur, 2003; Shaver et al., 2014).

The clinical picture of the population referred to our hospital is dominated by MPL, while LPL occurred in $10.2 \%$ of the dogs. According to the literature, lateral dislocation has been observed approximately in 5\% to $12 \%$ of dogs with PL, however, this was increased in large and giant breed dogs to reach $17 \%$ to $33 \%$ (Hayes et al., 1994; Arthurs and Langley-Hobbs, 2006; Alam et al., 2007; Shaver et al., 2014). 
Table 1. Distribution of lateral patellar luxation. Surgical management, complications, and outcome.

\begin{tabular}{ccccccccc}
\hline Cases & Breed & Sex & $\begin{array}{c}\text { Age } \\
(\mathrm{months})\end{array}$ & $\begin{array}{c}\text { Weight } \\
(\mathrm{kg})\end{array}$ & Grade & CrCL & Surgery & Complications \\
\hline $1^{*}$ & Poodle & F & 5 & 2.2 & 3 & Outcome & TTT, STP, CP & excellent \\
2 & Poodle & F $^{* *}$ & 36 & 3 & 3 & TTT, STP, TP & Seroma; implant migration \\
3 & Poodle & F & 7 & 5.2 & 3 & TTT, STP, CP & excellent \\
4 & Cavalier King & M & 48 & 7.9 & 1 & Yes & TTT, STP, FS & Seroma; implant migration \\
5 & Mixed-breed & M & 36 & 8.8 & 1 & & TTT, STP & excellent \\
6 & Pekingese & F & 84 & 6.8 & 2 & TTT, STP & excellent \\
7 & Pinscher & F & 132 & 2.3 & 3 & TTT, STP, TP & fair \\
8 & Pinscher & F & 12 & 4 & 2 & TTT, STP & good \\
9 & Yorkshire & F & 18 & 1.3 & 3 & TTT, STP, TP & Reluxation & Good \\
\hline
\end{tabular}

(TTT): Tibial tuberosity transposition; (STP): Soft tissue procedure; (CP): Condroplasty; (TP): Throcleoplasty; (FS): Fabellar suture.

* With bilateral patellar luxation. ** Neutered.

Most of dogs included were young at the time of examination, which is consistent with previous reports (Hayes et al., 1994; Arthurs and Langley-Hobbs, 2006; Kalff et al., 2014; Shaver et al., 2014). The young age was frequently associated to grade III or IV of lateral luxation due to congenital reasons as a result of underdevelopment of the trochlear groove. However, the disease may be diagnosed in older dogs, as low-grade luxations are usually asymptomatic in early stages (Kalff et al., 2014).

Surgical treatment was advised for all dogs with a diagnosis of PL. TTT may be the most important component of surgical treatment for dogs with PL since quadriceps malalignment is a key feature in the development of all grades of PL (Robins, 1990). A previous study evaluated the effect of the sole TTT without trochlear groove deepening in $91 \mathrm{dogs}$ with MPL, and concluded that trochlear groove deepening procedures are not always necessary, and patients that undergo these techniques should be carefully selected (Linney et al., 2011). Dog included in this report received adjunctive trochlear groove deepening when a clinical and radiographic evidence of shallow groove was observed.

Complications after surgical treatment of LPL have been previously reported, however one of the major limitation was the highly variable nature of surgical correction of LPL, which made impossible to draw conclusions about the effect of specific treatments (Shaver et al., 2014). The population reported here was managed with the same techniques (TTT and STP), with adjunctive procedures as needing, and our results support the effectiveness of the procedure that can result in definitive stabilization of the patella in its trochlear groove.

In the present case series, reluxation is the most important complication after surgical correction and occurred in $20 \%$ of stifle joints treated. Arthurs and Langley-Hobbs (2006) and Shaver et al. (2014) found respectively that $17 \%$ and $21.3 \%$ of stifle joints with LPL have reluxated, and this data is comparable with the percentage reported here. Kalff et al. (2014), accounting this complication in 6/65 of the dogs treated, reported the lower incidence of lateral reluxation. Minor complications included the migration of the pins and the following development of a seroma, and were easily managed through pins removal. Even though pin migration requires a minor surgical procedure for removing the Kirschner wires, it has been classified as minor complication (Kalff et al., 2014). Previous studies detected a correlation between body weight and a higher risk of complications and reluxation after corrective surgery (Arthurs and Langley-Hobbs, 2006; Gibbons et al., 2006; Cashmore et al., 2014). Because the dogs included are lightweight, probably stresses on stifle joints are lower, and the response to surgery may be better; furthermore the young age of affected dogs may play a central role in better recovery, due to the great tendency of tissues to have fast healing, and also for the absence of chronic degenerative joint lesions.

Shaver et al. (2014) stated that bilateral surgery during a single session was the only variable significantly associated with reluxation, accounting odds of reluxation 12.5 times higher than dogs that had unilateral surgery. We cannot confirm this data as most of the dogs were unilaterally affected, and the only one dog with a bilateral luxation was managed in double session surgery. Moreover, the limited number of stifles studied is a main limitation. Wangdee et al. (2013) evaluating the surgical management of MPL in Pomeranian dogs, established that grade II luxation had good outcome with a $100 \%$ success rate following surgery. Dogs with grade III luxation had recurrent PL in about $11 \%$ of the stifle joints treated; while dogs with grade IV luxation had higher rate of reluxation, accounting $36 \%$ of the dogs subjected to surgery, because varying degree of skeletal deformities. 
Most of the dogs included in this sample had positive outcome. Whereas our hypothesis about the two dogs that experienced fair outcome is that it may be more linked to the concurrent $\mathrm{CrCL}$ and pelvic injuries than to the PL.

The knowledge about LPL in the dog is still limited. The present report suggests that small breed dogs seems to be affected as well as medium, large and giant breed dogs. It can be concluded that previous studies analyzed populations with different prevalence of each breed and data obtained cannot be compared, LPL is a growing disease in small breed dogs and that LPL in small breed dogs is underestimated.

\section{References}

Alam, M.R., Lee, J.I., Kang, H.S., Kim, I.S., Park, S.Y., Lee, K.C. and Kim, N.S. 2007. Frequency and distribution of patellar luxation in dogs. 134 cases (2000 to 2005). Vet. Comp. Orthop. Traumatol. 20, 59-64.

Arthurs, G.I. and Langley-Hobbs, S.J. 2006. Complications associated with corrective surgery for patellar luxation in 109 dogs. Vet. Surg. 35, 559566.

Bound, N., Zakai, D., Butterworth, S.J. and Pead, M. 2009. The prevalence of canine patellar luxation in three centres. Clinical features and radiographic evidence of limb deviation. Vet. Comp. Orthop. Traumatol. 22, 32-37.

Cashmore, R.G., Havlicek, M., Perkins, N.R., James, D.R., Fearnside, S.M., Marchevsky, A.M. and Black, A.P. 2014. Major complications and risk factors associated with surgical correction of congenital medial patellar luxation in 124 dogs. Vet. Comp. Orthop. Traumatol. 27, 263-270.

DeAngelis, M. 1996. Patellar luxation in dogs. Vet. Clin. North Am. Small Anim. Pract. 1, 403-415.

DeAngelis, M. and Lau, R.E. 1970. A lateral retinacular imbrication technique for the surgical correction of anterior cruciate ligament rupture in the dog. J. Am. Vet. Med. Assoc. 157, 79-84.

Gibbons, S.E., Macias, C., Tonzing, M.A., Pinchbeck, G.L. and McKee, W.M. 2006. Patellar luxation in 70 large breed dogs. J. Small Anim. Pract. 47, 3-9.

Harasen, G. 2006. Patellar luxation: Pathogenesis and surgical correction. Can. Vet. J. 47, 1037-1039.

Hayes, A.G., Boudrieau, R.J. and Hungerford, L.L. 1994. Frequency and distribution of medial and lateral patellar luxation in dogs: 124 cases (19821992). J. Am. Vet. Med. Assoc. 205, 716-720.

Kalff, S., Butterworth, S.J., Miller, A., Keeley, B., Baines, S. and McKee, W.M. 2014. Lateral patellar luxation in dogs: a retrospective study of 65 dogs. Vet. Comp. Orthop. Traumatol. 27, 130-134.

LaFond, E., Breur, G.J. and Austin, C.C. 2002. Breed susceptibility for developmental orthopedic diseases in dogs. J. Am. Anim. Hosp. Assoc. 38, 467-477.

L'Eplattenier, H.F. and Montavon, P. 2002. Patellar luxation in dogs and cats: management and prevention. Comp. Cont. Edu. Pract. Vet. 24, 292300.

Linney, W.R., Hammer, D.L. and Shott, S. 2011. Surgical treatment of medial patellar luxation without femoral trochlear groove deepening procedures in dogs: 91 cases (1998-2009). J. Am. Vet. Med. Assoc. 238, 1168-1172.

Ness, M.G., Abercromby, R.H., May, C., Turner, B.M. and Carmichael, S. 1996. A survey of orthopaedic conditions in small animal veterinary practice in Britain. Vet. Comp. Orthop. Traumatol. 9, 43-52.

Nunamaker, D.M. 1985. Patellar luxation. In Textbook of Small Animal Orthopaedics, Eds., Newton, C.D. and Nunamaker, D.M.: Lippincott, Philadelphia, pp: 941-947.

Priester, W.A. 1972. Sex, size, and breed as risk factors in canine patellar luxation. J. Am. Vet. Med. Assoc. 4, 633-636.

Robins, G.M. 1990. The canine stifle joint. In Canine Orthopedics, Ed., Whittick, W.G.: Lea and Febiger, Philadelphia, pp: 693-760.

Roush, J.K. 1993. Canine patellar luxation. Vet. Clin. North Am. Small Anim. Pract. 23, 855-868.

Shaver, S.L., Mayhew, K.N., Sutton, J.S., Mayhew, P.D., Runge, J.J., Brown, D.C. and Kass, P.H. 2014. Complications after corrective surgery for lateral patellar luxation in dogs: 36 cases (2000-2011). J. Am. Vet. Med. Assoc. 244, 444-448.

Vasseur, P.B. 2003. Stifle joint. In Textbook of Small Animal Surgery, Ed., Slatter, D.S.: Saunders, Philadelphia, pp: 2090-2133.

Wangdee, C., Theyse, L.F.H., Techakumphu, M., Soontornvipart, K. and Hazewinkel, H.A. 2013. Evaluation of surgical treatment of medial patellar luxation in Pomeranian dogs. Vet. Comp. Orthop. Traumatol. 26, 435-439. 